\title{
Destins du deuil périnatal : un cas clinique
}

\author{
Luis Alvarez, Amina Yamgnane, \\ Giulia Disnan, Claire Squires
}

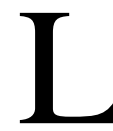

e parcours de Mme Martin à la maternité IPP-Necker nous amène à penser la clinique périnatale, à partir de la psychanalyse, dans ses dimensions intrapsychiques, relationnelles et institutionnelles. En effet, son histoire met en relief les fonctions de pare-excitation, de contenance et de mise en sens du pédopsychiatre de liaison en maternité et en pédiatrie auprès des patients, mais surtout auprès des collègues dans l'institution et notamment dans ses interstices (Bydlowski (a), 2002). Ce positionnement du pédopsychiatre à la maternité s'apparente, mutatis mutandis, aux points de vue de R. Cahn (1987) quant à la prise en compte des projections des composants clivés de la psyché de l'adolescent psychotique dans l'espace psychique des soignants, définissant ainsi une forme de «transfert institutionnel». Selon cet auteur, ce transfert sur l'institution possède une dimension davantage narcissique lorsque le contenu psychique projeté est fortement persécuteur. Dans ce cas, l'espace institutionnel prend la valeur d'un espace transitionnel, de la qualité duquel dépendra en grande partie l'issue du processus thérapeutique.

Luis ALVAREZ - Pédopsychiatre Adjoint du Service de Pédopsychiatrie de l'Institut de Puériculture de Paris.

Amina YAMGNANE - Gynécologue-obstétricienne à la maternité de l'Hôpital Necker Enfants-Malades (Paris).

Giulia DISNAN - Psychologue au Service de chirurgie viscérale pédiatrique de 1'Hôpital Necker Enfants Malades (Paris).

Claire SQUIRES - Pédopsychiatre, Institut Claparède (Neuilly s/s Seine) et Maternité de Port-Royal (Paris), Maître de Conférences à Paris VII. 


\section{LA MATERNITÉ: LE DERNIER REMPART.}

Le Dr. A. a rencontré Madame Martin, femme âgée de trente-trois ans, dans le Service de Grossesses Pathologiques de la Maternité IPP-Necker, à la demande des sages-femmes et du Dr. Y., son obstétricienne référente, dans le cadre de ses fonctions de pédopsychiatre de liaison. Sa grossesse gémellaire était prise en charge pour une menace d'accouchement prématurée, rebelle à tout traitement, à la 28ème semaine d'aménorrhée (SA). Elle était transférée d'une maternité niveau deux de la grande couronne parisienne devant l'imminence d'un accouchement à un si petit âge gestationnel. Cette patiente déconcertait et effrayait une équipe, pourtant habituée à l'univers de la maternité, par son agressivité, son ambivalence, son impulsivité, la labilité de son humeur, l'intensité de son angoisse, l'impossibilité d'entrer en contact avec elle, mais avant tout, par les propos très inquiétants qu'elle tenait au sujet des jumelles qu'elle portait et qui ont fait planer, parmi les soignants, le fantasme de l'infanticide : «elles me bouffent de l'intérieur... je le sens... je gonfle comme un ballon... ça va éclater... il faut les sortir de là... c'est soit elles, soit moi... les médecins ne comprennent pas... ils ne pensent qu'aux jumelles... je vais m'ouvrir le ventre ! ». L'intensité de l'ambivalence de Mme Martin, bien au-delà de celle qui accompagne la dynamique psychique habituelle de la femme enceinte (Alvarez et Golse, 2008), semblait avoir produit précocement un vécu gestationnel persécutif archaïque, de dévoration et sadisme oral et de rétorsion anale. Les premiers échanges avec le pédopsychiatre ont été marqués par sa méfiance et sa réticence. Une hospitalisation de plusieurs semaines était envisagée et elle n'a pu lier une véritable alliance thérapeutique qu'après avoir constaté que sa détresse ne faisait pas fuir une équipe prête, au contraire, à l'accueillir. Aide-soignante dans un hôpital de la banlieue parisienne, elle vivait dans la détresse l'éloignement vis-à-vis de son conjoint et de son milieu, du fait de cette hospitalisation. Elle expliquait ses propos inquiétants par «l'incompatibilité » entre son état de grossesse et sa «phobie des femmes enceintes ». Pour Mme Martin, cette incompatibilité était à l'origine de l'accouchement prématuré et de la mort d'un premier enfant, né à la $25^{\mathrm{e}}$ semaine d'une gestation qui se termina, à quelques jours de différence, exactement une année auparavant. À cette occasion, elleavait 
été prise en charge dans une autre maternité niveau trois. Elle se retrouvait, donc, hospitalisée, à la 28ème semaine d'une nouvelle grossesse très difficilement vécue, à un an de distance de la perte de Romain, son premier bébé : "À l'autre maternité... ils n'ont rien fait pour le sauver... ils s'en foutaient... ils étaient là à me parler... mais Romain est bien mort... je leur en veux...», puis «ici... c'est pareil... tout va se passer comme l'année dernière...». Le délai souhaitable pour concevoir un autre enfant après la mort périnatale suscite des discussions. D'une part, les auteurs craignent que les parents ne s'accordent pas assez de temps pour commencer une autre grossesse ou qu'une grossesse trop rapprochée inhiberait le deuil et pourrait conduire à une idéalisation, voire à un rejet de l'enfant, et à une maltraitance selon Lewis (1979). Bowlby dans Attachement et perte (1984) suggère d'attendre un an. D'autre part, d'autres auteurs considèrent qu'une grossesse suivante contribue à alléger les conséquences du deuil (Jensen et Zahourek, 1972; Wolf, 1970). Ces deux attitudes, en apparence opposées, témoignent de l'embarras des cliniciens. D'ailleurs, les conseils sont toujours mal reçus quoi qu'il arrive. La perte d'un enfant au cours de la grossesse constitue un événement non négligeable ; les réactions à cette mort in utero grèvent toujours le déroulement de la grossesse suivante quel que soit le délai à concevoir. Les différentes études concernant le délai utile à surmonter la mort in utero et à concevoir un autre enfant sont donc contradictoires. Différencier les mères pour qui la grossesse suivante aide à résoudre la problématique de deuil et les mères qui l'évitent s'avère difficile (Zeanah, 1989) mais souvent la prise en compte de la mort in utero n'est pas faite lors d'une grossesse ultérieure (Squires, 2002). Et c'est bien ce qui semble s'être passé pour Mme Martin.Le partage de ces éléments traumatiques, et notamment la réalisation du calcul inconscient (Bydlowski (b), 2002) autour de la coïncidence des dates des grossesses autorisait la co-construction d'un cadre psychothérapique et d'hospitalisation respectueux des besoins de cette patiente. Ainsi, le Dr. A. et Mme Martin se sont mis d'accord pour fixer une fréquence bihebdomadaire à leurs séances et pour resituer aux soignants quelques éléments de son histoire, afin de leur permettre de mieux s'ajuster à sa problématique. Lors de cette restitution, en présence du Dr. Y., l'équipe a pu entendre que sa compétence et la qualité de son travail, fortement questionnées et 
attaquées par Mme Martin, n'étaient pas l'enjeu majeur de sa prise en charge multidisciplinaire. Mme Martin ne pouvait que réactualiser sur la scène institutionnelle les événements traumatiques qui l'habitaient et qui ont donné un sens déterminé à la manière dont elle avait fait le récit de sa grossesse précédente. À l'issue de cette concertation entre soignants, il a été décidé que les Drs. Y et A. et la sage-femme référente des grossesses pathologiques allaient se rendre ensemble au chevet de Mme Martin pour lui proposer quelques modifications du cadre de l'hospitalisation. Ce discours consensuel porté conjointement par l'obstétricienne, la sage-femme et le pédopsychiatre, signifiait à Mme Martin qu'elle avait affaire à une équipe multidisciplinaire contenante, limitante, cohérente, différenciée, capable de cohésion et de remise en question. De cette manière, l'obstétricienne et le pédopsychiatre commençaient à constituer pour cette patiente un véritable couple parental thérapeutique, non-clivable, assumant la bisexualité psychique. Cette capacité des soignants d'allier à chaque instant la fonction maternelle et la fonction paternelle, selon les besoins des patients, donne au cadre sa qualité malléable. La malléabilité fait éprouver aux patients que le cadre coconstruit est en mesure de se laisser modeler par leur complexité, gardant leur empreinte sans se déformer, se pervertir ou se détruire (Milner, 1990). À l'intérieur du cadre de l'hospitalisation, le Dr. Y. et la sage-femme référente des grossesses pathologiques allaient proposer une stratégie thérapeutique sur le plan obstétrical, adaptée aux besoins de la mère et des jumelles, stratégie qui visait à donner du sens au déplacement de fragments de l'histoire de Mme Martin sur le lieu de l'hospitalisation. Dans cette logique, la sage-femme référente allait proposer à Mme Martin des rencontres aux moments clefs de la vie institutionnelle, troquant le conflit par sa présence et des échanges. Elle allait prendre un soin particulier « à faire le pont entre Mme Martin et les soignants », notamment lors des changements d'équipe. De cette manière, la maternité et ses soignants sont devenus pour Mme Martin, le dernier rempart face à l'irruption d'une angoisse mettant en question la cohésion de son appareil psychique. Lors des consultations prénatales, l'obstétricien peut être dérouté par la confusion chez la future mère, dans la mesure où les représentations de mort se superposent à l'investissement de la grossesse actuelle. Les femmes apparaissent alorsparticulière- 
ment réservées, peu exubérantes, sombres, accablées, préoccupées... Les mères sont aussi inquiètes, avides de questions parfois envahissantes pour le médecin. À travers ces comportements inhabituels des mères, la demande est le plus souvent formulée en un souhait de réassurance, alors que le corps médical a «échoué » antérieurement, lors de la grossesse précédente. Une défiance à l'égard du médecin est fréquente; elles le testent par leurs questions qui tournent toujours autour de la confiance qu'elles aimeraient bien retrouver. L'obstétricien n'a pas toujours la possibilité de leur donner des «causes» expliquant la mort in utero et il ne peut garantir le futur. Lorsque les parents ont changé d'hôpital, environ dans la moitié des cas, l'équipe obstétricale est mise en demeure d'être plus performante que la précédente. Le clivage entre «bonne équipe obstétricale» et «mauvaise équipe obstétricale» vient souvent à la place d'une prise en considération des affects dépressifs liés à la mort in utero. En établissant un lien avec les soignants précédents à qui il a demandé un bilan détaillé des faits médicaux, l'obstétricien a la lourde charge d'à la fois justifier, s'il le peut, la prise en charge précédente, ou bien d'en reconnaître les écueils. Il lui faut restaurer une certaine confiance vis à vis de l'obstétrique mais aussi prendre une certaine distance avec l'ancienne équipe, afin de constituer ses propres marques et circonscrire le champ de ses interventions. L'obstétricien peut être pris dans ce double mouvement de défiance des parents et de demande de réassurance. En tant que médecin, il doit être attentif à tout, en prêtant attention à tous les symptômes faisant craindre une alerte (une contraction, une douleur), en multipliant les examens, en proposant une écoute et en montrant que l'ensemble de l'équipe sera prête à réagir, le cas échéant. La réponse porte d'ailleurs plus souvent sur l'assurance de sa capacité à réagir dans le domaine médical que sur une restauration de la confiance de la parturiente à mener à bien cette grossesse jusqu'à son terme. De la capacité de l'obstétricien à faire en sorte que cette grossesse actuelle soit vécue de façon différente de la précédente, dépendra l'issue heureuse de cette nouvelle gestation. Pour cela, même la réticence des mères, leurs exigences, leur peine, voire leur agressivité, leur profonde culpabilité, devront être au moins prises en considération. Les médecins se trouvent parfois induits à des contre-attitudes inhabituelles, par excès d'interventions, gagnés eux-aussi et malgré eux par l'inquiétude des mères. 


\section{LA PHOBIE DES FEMMES ENCEINTES.}

À la séance suivante, le Dr. A. a demandé quelques précisions au sujet de la situation paradoxale dans laquelle Mme Martin semblait se trouver : "Vous êtes enceinte... et vous dites que vous avez la phobie des femmes enceintes...». Elle affirmait que son désir d'enfant était sincère, malgré la surprise qu'il générait en elle. En effet, Madame Martin provenait d'un milieu très marginal et, à l'adolescence et au début de la vie adulte, elle avait «frôlé » la toxicomanie et la prostitution : «... je viens de loin... je n'aurais jamais cru qu'un jour je désirerais avoir un bébé...». La rencontre avec Monsieur Martin, son aîné d'une dizaine d'années, avait marqué un tournant décisif dans sa vie. Il avait résisté aux assauts de la haine, à ses tentatives de clivage, à ses angoisses d'abandon, à son impulsivité, à son ambivalence et à ses passages à l'acte. Cette vie de couple avait permis à Mme Martin de sortir de la marginalité, de réparer son narcissisme, de reprendre tardivement ses études et, enfin, de désirer un enfant. Mais, comment concilier le désir d'enfant avec une phobie de femmes enceintes? En effet, la vision d'une femme enceinte déclenchait chez cette patiente, depuis l'adolescence, une forte bouffée d'angoisse, avec une importante composante somatique, événement qui se soldait, parfois, par un évanouissement : «À l'école d'aidessoignantes... j'ai triché... une copine a fait une partie du stage en salle de naissances à ma place... puis... Il a fallu que j’y aille... je me cachais derrière les draps... puis un jour... j'ai dû assister à un accouchement... je suis tombée dans les pommes... j'ai dit que je n'avais pas pris de petit déjeuner». Madame Martin voulait un enfant sans passer par la grossesse. Ainsi, lorsqu'elle était enceinte, les changements physiologiques de la grossesse et surtout les mouvements fœtaux suscitaient une grande angoisse et alimentaient des fantasmes très archaïques de morcellement, d'éclatement, de perte de la cohésion corporelle, d'envahissement, de dévoration intérieure. Lorsqu'une grossesse multiple est survenue, cette expérience somatique et ces fantasmes s'avéraient insupportables : «... je suis doublement pleine...». Ces productions fantasmatiques étaient à la base des propos inquiétants qu'elle livrait aux sages-femmes et aux obstétriciens. Le Dr. A. s'est aperçu que l'intensité de l'angoisse de Mme Martin ne lui permettait pas de prendre contact avec la perte de son bébé de 
l'année dernière. L'expérience de Romain, bien qu'advenue, ne pouvait pas s'inscrire. Ainsi, elle semblait piégée dans l'instant, pétrifiée à mesure qu'elle assistait, presque de manière intransitive, au déploiement de ses fantasmes et à perte de cohésion de sa vie psychique, comme si la grossesse précédente constituait un non-évenement, vécu comme une hallucination négative lors de la grossesse suivante. À presque deux semaines d'hospitalisation, les liens entre Mme Martin et l'équipe s'étaient apaisés et un lien de confiance s'était établi avec la sage-femme référente. Le cadre co-construit semblait donc la contenir, lui permettre donner du sens aux affects ravageurs qu'elle ressentait accentués par la culpabilité liée à la mort in utero précédente et limiter l'effet ravageur de ses angoisses. Le début de la psychothérapie et son association à un traitement anxiolytique avait, quelque peu, soulagé l'angoisse et un hypnotique diminuait une insomnie persistante. La sédation de l'angoisse était suivie d'un retour des fragments du passé de Madame Martin, parmi lesquels, ceux de son enfance. Elle évoqua, alors, les mauvais traitements et la négligence d'une mère alcoolique, ayant «frôlé » la prostitution, qui la confia, de même que sa sœur cadette, à sa tante, lorsqu'elle comptait six années. Elle avait pu nouer ainsi des liens solides avec la sœur de sa mère. Une remémoration majeure de cette époque a marqué un pas conséquent dans la psychothérapie, contemporaine d'un amenuisement des contractions utérines. Vers l'âge de neuf ans, sa tante l'avait amenée à l'hôpital pour rendre visite à sa mère, à qui les médecins venaient d'amputer ses deux jambes dans les suites d'une artérite «négligée ». À cette occasion, elle avait regardé longtemps les moignons de sa mère. Celle-ci est morte peu après à l'âge de trente-trois ans, des complications de l'artérite. Cette mise en mots semblait annoncer l'énonciation d'un scénario narcissique (Manzano J., Palacio-Espasa F., Zilkha N, 1999) se précipitant sur cette deuxième grossesse. L'évocation de ce souvenir d'enfance traumatique déclencha un épisode somatique grave lors de la seconde grossesse témoignant des failles narcissiques de la personnalité fragile de Mme Martin, décompensée lors d'une situation d'angoisse intense favorisée par la situation de régression thérapeutique. Il me semble qu'on peut parler à propos de Mme Martin de dépression essentielle, telle que l'envisage Pierre Marty. Souvent précédée d'angoisses diffuses liées à l'afflux de mouvements instinc- 
tuels non maîtrisés parce que non-élaborables (évoquant la névrose d'angoisse freudienne), il s'agirait d'un état archaïque de débordement, n'ayant aucune valeur de signal d'alarme apte à mobiliser les défenses mentales. Le Moi submergé montre sa faiblesse défensive, l'insuffisance de ses recours, son défaut d'organisation, sa désorganisation. Aucun travail de liaison ne peut s'accomplir. Les patients viennent pour des maladies somatiques, résultantes de conflits physiologiques, correspondant à des niveaux d'organisations défensives de type régressif, préétablies autour de fixations de diverses origines. Dans ces cas, Pierre Marty explique que l'aide médicale classique est particulièrement efficace. La maladie peut imposer des changements dans la vie du patient et de l'entourage. Il peut survenir chez la patiente un sursaut narcissique sur lequel on peut alors tenter de prendre assise psychothérapeutiquement.

\section{LA MISE EN CORPS DE L'OBJET PERDU.}

La remémoration de cet épisode par Mme Martin s'est accompagnée, dans les jours qui ont suivi, de sa mise en corps par l'apparition d'une thrombose veineuse des deux jambes. Simultanément, elle s'est mise à tricoter et à chercher des prénoms de fleurs pour ses jumelles, elle-même portant un prénom de fleur, comme pour symboliser la présence de ces femmes aimées et, néanmoins, si absentes. En outre, le souvenir de la mort de sa mère l'avait amenée au souvenir de la mort de sa tante lorsqu'elle avait douze ans. Ce décès l'avait plongée dans un profond désarroi et suscita en elle des sentiments d'abandon et d'angoisse. Elle avait été confiée par le juge à sa cousine Alice, fille de la défunte, jeune femme assumant un enfant en bas âge, peu disponible pour se pencher sur la situation d'une préadolescente à la dérive. Mme Martin poursuivait son récit au cours des séances : aux prises avec un profond sentiment d'abandon, elle avait cherché consolation dans les sollicitations qu'un ami de sa tante, d'au moins vingt ans son aîné, lui faisait et débuta ainsi «ma première relation de femme...» : «Alice... avec son mari et sa vie... n'avait pas le temps de remplacer ma tante... je me sentais seule... malheureuse... Alors Joseph a commencé à venir à la maison... on parlait de ma tante... ça me faisait du bien... puis 
il me faisait de petits cadeaux... enfin quelqu'un qui s'intéressait à moi... puis il a commencé à me toucher... je n'en savais rien... puis ce n'était pas désagréable...». Cette relation, secret de polichinelle pour sa cousine, s'est soldée par une grossesse, à l'âge de treize ans. Mme Martin ne gardait que des souvenirs confus d'un avortement clandestin, organisé par cet homme, qui s'est suivi de très douloureuses contractions : "On est entrés par l'arrière de l'hôpital... je crois que c'était un hôpital... je me suis allongée et Joseph me donnait la main... puis ça a commencé... comme ça, à vif... j'avais mal... comme si on me déchirait à l'intérieur... après... à la maison... j'avais mal et je saignais... je ne pouvais rien dire à personne... je pensais que $\mathrm{j}$ 'allais crever... Joseph m'avait fait promettre de ne rien dire... j'aurais fait n'importe quoi pour qu'il reste avec moi ! ». Après cet épisode, son amant l'a également abandonnée : «Je détestais les gens... rien de bon ne pouvait venir d'eux... alors... j'ai fait les quatre-cents coups... jusqu'à ce que je rencontre mon mari... sans lui... j'aurais crevé il y a bien longtemps...». Madame Martin a pu alors situer l'apparition du premier épisode de la phobie de femmes enceintes lorsque, peu de temps après l'avortement, sa cousine Alice, avait débuté une nouvelle grossesse : «Je vomissais plus qu'elle... je ne sais pas si elle a compris... je ne pouvais pas la voir... alors j'ai fugué... je traînais ici et là... puis le juge m'a mise dans un foyer... ma sœur est restée avec Alice... c'est pour ça qu'on ne s'entend pas...». L'exhumation de cette série de remémorations de la présence maternelle chaotique, de la tante vite disparue, de l'amant incestuel fuyard, de la grossesse adolescente avortée a été contemporaine d'un arrêt des contractions et d'une disparition de l'angoisse. Alors que la première grossesse avait condensé les éléments mortifères du passé de Mme Martin et peut-être contribué à son interruption, la seconde grossesse se différenciait progressivement de la première grâce à ce travail thérapeutique de deuil et de subjectivation. Madame Martin investissait de ses rêveries ces deux enfants et nouait des liens de qualité avec l'équipe soignante. C'est alors qu'elle a partagé le vécu de ces deux semaines de psychothérapie : «... je ne pensais jamais à tout ça, depuis un bout de temps... je vous l'ai dit parce que je suis enceinte et parce que je sais qu'après l'accouchement je ne vais plus jamais vous revoir... après l'accouchement je ne vais plus jamais en parler...». 


\section{LA GROSSESSE DE MONSIEUR MARTIN}

Pour les soignants de la maternité, le grand absent était $\mathrm{M}$. Martin. Travailleur indépendant, il ne rendait visite à son épouse que certains soirs et était décrit par les équipes de nuit comme un homme discret, avenant et faisant preuve d'une grande sollicitude à l'égard de Mme. Un matin, le Dr. A. a découvert Mme Martin très affairée avec des échantillons de papiers peints dans les mains : «C'est pour mon mari... il est en train de faire la chambre des gamines... on avait trouvé un modèle qui nous plaisait... mais hier il m'a dit qu'il était épuisé... alors... il faut en choisir un autre... ce soir il va passer... et je dois lui dire ceux qui me plaisent...». Pour la première fois, les angoisses archaïques cédaient la place à la tristesse et à la nostalgie, attestant de ses tentatives de liaison des fantasmes réinvestis par la grossesse : «J'aime beaucoup ma maison... mon mari me manque... je me demande si notre vie ne pas trop changer avec l'arrivée des jumelles... je veux dire... est-ce qu'il va m'aimer comme maintenant?». L'apparition de cette rivalité avec les jumelles, d'allure névroticoœdipienne, et de cette scénarisation plus secondarisée de la problématique d'abandon, accompagnée d'une tonalité affective plus fluide permettant une certaine dépressivité, semblait indiquer l'entrée dans la perlaboration, puis dans un processus de construction d'un nouveau récit de son passé et des nouvelles virtualités avec ses enfants et son mari : « Je l'ai connu quand j'étais dans la déch... j'avais dans les vingt ans... j'étais presque SDF... je ne comprenais pas ce qu'il me voulait... puis il me saoulait avec son air de moine... sage... mais alors... je lui ai en fait voir de toutes les couleurs... des disputes... je partais au milieu de la nuit... je le trompais... puis... il restait là... j'ai compris qu'il m'aimait... moi... la merde... il m'aimait... je n'en revenais pas...». Mme Martin a été la première surprise par les effets de ces constatations : «Là... je me suis mise à flipper... s'il m'aimait... après tout ce que je lui avais fait... je pouvais le perdre avec mes conneries... qu'est-ce que j'avais été conne... alors je lui ai proposé qu'on se marie... il a accepté... tout de suite... on est mariés depuis onze ans... j'ai arrêté mes conneries...». Le clivage, l'intrusion, l'identification projective, les angoisses d'abandon et la persécution ont à nouveau refait surface (sont revenus au galop) lorsque le Dr. A. lui propose de convenir d'un rendez- 
vous avec M. Martin : « Mais qu'est-ce que vous voulez lui dire ? Il sait déjà tout ce que je vous ai dit... il m'a connu à cette époque... vous les psys... vous aimez remuer la merde ! Ma place est ici à l'hôpital... pour les gamines... ça c'est ma grossesse... la place de mon mari est à la maison... il prépare la chambre des gamines... puis, il fait tout ce que je me peux pas faire en étant ici... sa grossesse à lui c'est la maison! ». Ne tentait-elle pas ainsi de le protéger de ses projections pathologiques en lui attribuant également un rôle maternel qui lui avait tant manqué ? Le recours à un fonctionnement pseudonévrotique, du fait de la restauration narcissique vécue avec son mari, avait permis à Mme Martin de s'extraire de la répétition ravageuse des principaux scénarios traumatiques de sa pathologie narcissique. Néanmoins, l'équilibre précaire de cet agencement défensif ne pouvait que tenir à distance les exigences de ses fragilités, comme l'atteste le retour en puissance des fantasmes archaïques du fait de la grossesse.

\section{PARTIR C'EST MOURIR UN PEU}

$\mathrm{Au}$ bout de trois semaines d'hospitalisation, l'évolution satisfaisante de Mme Martin ne nécessitait plus une hospitalisation dans une maternité de niveau trois raison pour laquelle Mme Martin demandait son transfert vers la maternité la plus proche de son domicile. Au fait de la problématique narcissique et abandonnique de cette patiente, les Drs. Y. et A. ont estimé nécessaire de «construire la sortie » à l'occasion d'une rencontre avec le couple. Mme Martin était rassurée d'entendre le souci du Dr. A. de préserver son intimité et de ne rien dévoiler à son mari de ce qu'elle lui avait confié. $\mathrm{M}$. Martin a pu être invité, en tant que mari et père, à envisager la meilleure manière d'organiser son transfert. En présence de son mari, la patiente adoptait une attitude régressive :

- Mme Martin était suivie par un obstétricien d'une maternité niveau deux où elle désirait accoucher. Elle savait que le Dr. Y. avait pris soin de tenir informé son collègue du déroulement de l'hospitalisation et de ce qu'elle avait permis de comprendre à ses difficultés.- Dans un deuxième temps, Mme Martin est apparue rassurée lorsque le Dr. Y. lui a réitéré la disponibilité de l'ensemble de l'équipe de la maternité IPPNecker «en cas de besoin...» et lui a signifié sa sollicitude si 
elle souhaitait prendre rendez-vous en ambulatoire.- Pour sa part, le Dr. A. a souligné le fait que le travail entamé à la maternité pouvait se poursuivre en ambulatoire, dans le cadre de sa consultation pédopsychiatrique. Aussi, il lui a demandé si elle jugeait utile qu'il prenne contact avec la psychologue de la maternité où elle devait accoucher.- M. Martin jouait un rôle apaisant et organisateur pour sa femme et s'est avéré un allié précieux pour la poursuite de la prise en charge.- Dans le souci d'éviter un transfert à la veille d'un week-end, la sortie a été prévue pour le début de la semaine suivante, complétant ainsi une vingtaine de jours d'hospitalisation. Le Dr. A. avait proposé un rendez-vous pour le milieu de la semaine et laissé au couple la possibilité de contacter son secrétariat « en cas de besoin».

Une fois toutes ces précautions prises, Mme Martin a fait des émotifs adieux aux soignants, avec remise de boîtes de chocolats et promesses de faire-part. Tout ce cérémonial du départ n'a pas permis à Mme Martin de quitter la maternité IPP-Necker. Elle a accouché de deux petites filles en bonne santé, la veille de son départ, à la 33ème semaine de gestation. Ses enfants ont dû séjourner brièvement dans le service de néonatologie.

\section{DEVENIR MÈRE}

L'accouchement de Mme Martin a été décrit avec étonnement par l'équipe de la salle de naissances: «Elle était comme ailleurs... elle nous parlait... suivait les événements... mais comme à distance... elle a eu sa péridurale... on s'attendait à ce qu'elle soit angoissée... on dirait qu'elle était shootée...». Mme Martin en a fait un récit laconique : «Je ne m'en souviens pas... je n'ai pas eu très mal... j'avais peur d'avoir mal... il faillait que ça se fasse... puis dès qu'on m'a dit que Rose et Marguerite allaient bien... c'était fini ». Ce récit s'apparentait à une expérience de dépersonnalisation, renforcée peut-être par une anesthésie péridurale fortement dosée. En néonatologie, Rose et Marguerite évoluaient de manière satisfaisante. Les Drs. Y. et A. ont eu à peine le temps de restituer aux pédiatres les grandes lignes de la prise en charge de Mme Martin et de ses modalités relationnelles. Dans un premier temps, Mme Martin s'est montrée ralentie, presque triste mais 
affichant une thymie émoussée. Devant l'équipe qui l'accompagnait à rencontrer ses petites filles Mme Martin avait une attitude régressive, docile, comme si elle assistait à un cours. Les puéricultrices décrivaient l'attitude figée de Mme Martin à l'occasion du «peau à peau ». Son comportement avec les bébés semblait dépourvu de rêverie, distant, opératoire. $\mathrm{M}$. Martin, incarnant la fonction paternelle, avait le souci de la mère et des filles, veillait à garantir la continuité entre elles et soutenait les compétences maternelles de son épouse. En sa présence, Mme Martin était moins figée, plus authentique avec les enfants. Au bout de cinq jours d'hospitalisation, un transfert vers le service de néonatologie le plus proche du domicile était envisagé. Le Dr. A. a rencontré Mme Martin et Rose, lorsque Marguerite faisait l'objet de soins. Rose dormait et sa mère, à ses côtés, paraissait perdue dans ses élaborations dépressives. Son fonctionnement psychique s'approchait davantage de celui de la fin de la grossesse que de celui du post-partum immédiat : " Je pense à ma tante... qu'aurait-elle dit des jumelles ? Elle aurait été fière de me voir aujourd'hui...». Mme Martin accordait donc aux jumelles le pouvoir de la dédommager et de dédommager sa tante des vicissitudes de l'histoire familiale. Aussi, les premiers éléments cliniques d'une dépression du post-partum se manifestaient : «L'accouchement m'a laissé comme vidée... je n'ai pas beaucoup d'énergie... je donne tout ce que je peux aux petites... mais je suis fatiguée...». Enfin, les fragilités narcissiques se manifestaient, avec leur cortège d'identification projective, de clivage et d'intrusion d'angoisses de différenciation, d'identifications projectives sur le personnel soignant, d'idéalisation de la fonction maternelle et d'auto-déqualification avec demande de réassurance : «J'ai très peur quand je suis seule avec elles... elles me demandent tant de choses... puis, je me sens observée par le personnel... je ne peux pas être naturelle... puis les gamines... surtout Marguerite... elles sont tellement petites... elles attendent tellement de moi... Elle ne veut pas manger avec moi Marguerite... avec son père elle mange ... mais pas avec moi... je ne sais pas si je suis à la hauteur », «C'est pour ça que j'ai arrêté l'allaitement... tirer le lait... c'était épuisant... puis leur donner le biberon... l'une puis l'autre... Avec Rosa... ça pouvait aller... mais avec Marguerite... c'était dur...». Avec l'accord du couple et devant l'imminence du transfert des bébés vers un lieu de soin proche de leur 
domicile, le Dr. A. a mis en place un traitement antidépresseur et prolongé la médication anxiolytique. Aussi, il a pris contact avec les pédopsychiatres et psychiatres d'adulte rattachés à l'hôpital qui accueillait Rose et Marguerite, en vue d'une reprise sur place des soins psychiques. En effet, Mme Martin comprenait qu'elle avait besoin que des soignants l'aident à entrer en contact avec ses enfants et que la distance entre le domicile et le site IPP-Necker ne rendait pas possible la poursuite de la prise en charge initiée pendant la grossesse. Les collègues qui ont pris le relais des soins ont donné quelques nouvelles de Mme Martin et de sa famille aux Dr. Y. et A. La mère a connu une évolution lente d'un véritable épisode dépressif se prolongeant jusqu'à l'âge de sept mois des bébés. Rose et Marguerite avaient connu un court séjour en néonatologie, suivi d'une prise en charge en hospitalisation à domicile, par la PMI et par l'intersecteur de pédopsychiatrie. À leur entrée en crèche elles présentaient un développement satisfaisant. Néanmoins, Marguerite a présenté précocement quelques symptômes anorexiques suivis de coliques et d'un reflux qui ont disparu à l'entrée en crèche. M. Martin continuait à veiller sur toute sa famille.

\section{CONCLUSION}

Cette vignette clinique illustre le fait que l'expérience somatique de la grossesse, avait apporté des excitations ayant, pour cette patiente, valeur de répétition du trauma sur la scène du corps. Ces excitations venaient investir les représentations de tout un ensemble d'événements qui, traumatiques, avaient participé à la fondation de son narcissisme. En effet, l'intensité de la répétition laissait l'appareil psychique dans l'impossibilité de lier ces représentations d'événements à l'ensemble de représentations du Moi et d'investir la grossesse de son narcissisme, ce qui apparentait le processus à une dépression essentielle. Ce récit clinique nous montre les maintes tentatives d'intégration que le Moi exerçait sur ces représentations d'événements, transformées en véritables " corps étrangers » (Rosenberg, 1997) pour le Moi de la patiente. D'abord, il est pertinent d'observer que ces tentatives de liaison ont été faites sur le mode de l'identification à trait unique : la mère et la fille ont « frôlé» la prostitution, la mère était alcoolique, la fille a 
eu recours aux psychotropes. Nous supposons que les liens que cette patiente avait noué avec sa tante pouvaient avoir un effet de réparation narcissique, comme l'atteste la vie de couple qu'elle su établir sur ce mode. Ces processus identificatoires correspondent à des actions qualifiables par les adjectifs de narcissiques secondaires. Également, la conception des deux grossesses était marquée par la possibilité d'accéder à des retrouvailles narcissiques avec sa mère par la répétition du traumatisme psychique : la mise à mal du corps (de sa mère, de ses enfants, d'elle-même), la rencontre avec la mort (de sa mère, de son premier enfant), dans le même lieu (l'hôpital), au même âge (les trente-trois ans de la mère et de la fille, l'accouchement à la 33ème semaine). En outre, il paraît pertinent de reconnaître à l'œuvre l'agissement une identification à trait unique dans le jeu de miroir entre les jambes de la mère et les jambes de la fille (amputation pour l'une, phlébite pour l'autre), véritable issue psychosomatique des retrouvailles avec les traces laissées par l'objet perdu. Cet épisode somatique suivant un trajet imaginaire comparable pour la mère et pour la fille n'est pas sans évoquer la patiente de Freud, Elisabeth von R., atteinte d'une paralysie hystérique des jambes après avoir soigné son père malade et posé les jambes de celui-ci sur ses propres cuisses. Mais ici, la somatisation diffère des conversions hystériques car le corps somatique est vraiment touché, tandis que pour l'hystérie, c'est le corps imaginaire, fantasmé qui est sollicité. Freud décrit le saut du psychique dans le somatique dans la névrose obsessionnelle de l'homme aux rats. On a distingué la conversion hystérique où le symptôme est l'expression dans le corps d'un conflit, d'un fantasme qui ne peut se dire, de la somatisation où le corps est vraiment atteint Valabrega parle de conversion généralisée hors de l'hystérie pour évoquer ce saut du psychique dans le somatique (Fantasme, mythe, corps et sens, 1980, Payot, Paris). La conversion psychosomatique et son caractère traumatique semblent ici faire échec à la conception d'un fantasme. Valabrega élargit la notion de la conversion aux voies de la sensorialité et du système neurovégétatif, Joyce Mac Dougall parle de conversion pré-génitale et C. Dejours d'un choix d'organe lié à un conflit pré-génital. Néanmoins, ces esquisses de rapprochement des imagos maternelles par le narcissisme secondaire, n'étaient pas dépourvues d'ambiguïté ou d'angoisse. Elles ne pouvaient conduire qu'à la répétition 
du traumatisme. Par ailleurs, ces rapprochements étaient aussi l'occasion d'agir la haine à l'encontre d'une femme qui n'a offert qu'un lieu maternel dévasté. Cet agissement de la haine ne pouvait que se retourner sur elle-même, son Moi étant l'endroit où le narcissisme secondaire avait fixé ces identifications. Ainsi, Mme Martin ne pouvait pas assumer une filiation à sa mère et, simultanément, elle ne pouvait pas cesser de la chercher. Ces jeux identificatoires ne traduisent autre chose que la nécessité pour le Moi de garantir son affirmation et sa conservation, comme le signale A. Green (1983) dans un de ses ouvrages sur le narcissisme. Ces regards doublés par des miroirs cachaient un autre traumatisme, qui était également représenté par ce scénario de maternité : la mise à mal d'une grossesse. La perte répétée des objets maternels, d'abord sa mère, puis sa tante, la renvoya à une relation sexualisée avec un homme de vingt ans son aîné. Cette relation se solda par une première grossesse et un avortement clandestin dont elle gardait le souvenir des contractions douloureuses et la phobie des femmes enceintes. Ainsi, l'introduction de ce nouvel événement vient compléter la chaîne associative et dévoiler les métamorphoses métaphorique et métonymique de ce scénario où l'enfant est mis à mal comme elle a été mise à mal. $\mathrm{Au}$ moment où elle allait dépasser l'âge de la mort de sa mère, la grossesse peut se comprendre comme un dernier essai de rapprochement par l'identification à une mère déstructurante et comme une tentative de filiation par la féminité. Cette recherche de l'objet perdu dans la répétition du traumatisme est venue résonner avec un autre traumatisme : la grossesse et l'avortement clandestin, lorsqu'elle comptait treize années. En effet elle « est tombée enceinte », à la suite d'une longue chute, marquée par l'impossibilité d'établir une identification structurante avec sa mère et la perte de sa tante, avec qui elle avait pu constituer ce que S. Freud qualifiait d'identifications fondatrices. Celle-ci était ainsi une grossesse d'horreur, où elle perdait, où elle tuait, de nouveau, des enfants dans des contractions douloureuses. La grossesse était, donc, ce compromis impossible entre les retrouvailles de l'objet perdu et la haine de l'objet par la construction d'un scénario où confluaient plusieurs maillons de chaînes associatives. Sur un autre plan, l'expérience de dépersonnalisation de l'accouchement, la persistance du régime psychique de la grossesse dans le postpartum immédiat, la rencontre laborieuse avec les bébés et 
l'agissement du clivage et des identifications projectives pathologique notamment sur l'une des jumelles viennent nous rappeler les failles et les blessures de la pathologie narcissique de Mme Martin, dont une apparence pseudo-névrotique équilibrait, en dehors de la grossesse, un fonctionnement dans le registre état limite. Toutes ces lignes de tension ont conduit à la précipitation d'un véritable épisode dépressif pendant les sept premiers mois de vie des enfants, issue connue dans le postpartum des mères porteuses de pathologies du narcissisme. Enfin, le développement des filles paraît satisfaisant, constat qui vient confirmer la notion que les effets délétères sur la construction des enfants des états dépressifs maternels et des discontinuités interactives, propres aux mères états limites, ne se manifestent qu'en absence de tiers. Dans le cas de Rose et Marguerite, le principal tiers est incontestablement leur père. Aussi, les interventions précoces et dans la durée d'obstétriciens, pédiatres, pédopsychiatres, puéricultrices, ainsi que le mode de garde collectif ont fourni un tiers soignant de qualité. Néanmoins, les identifications projectives pathologiques dont Marguerite a fait l'objet se sont associées à des issues psychosomatiques passagères. Celle de Mme Matin était donc une famille à risque, nécessitant une présence soignante structurante dans la durée, dans une logique de prévention des troubles précoces du développement, de soins de la parentalité et de prise en charge psychothérapeutique pour la mère. Le parcours de cette famille fait ainsi l'éloge de la lenteur, qui est celle des cliniciens du premier âge, d'autant plus que les retrouvailles avec les objets traumatiques parentaux tendent à accélérer le temps, à cliver la réalité et à amorcer un nouveau terme de la répétition.

\section{BIBLIOGRAPHIE}

ALVAREZ L. et GOLSE B. (2008), Les compétences des parents, La Psychiatrie du Bébé, Paris, PUF, pp. 34-45.

BOWLBY J., (1978), Attachement et perte: vol.1, l'attachement, vol.2, la séparation, perte, tristesse et dépression, vol 3, Paris, PUF, le fil rouge, 1984.

BYDLOWSKI M. (a 2002), Le travail psychanalytique en Maternité, La dette de vie. Itinéraire psychanalytique de la maternité. Paris, PUF, pp. 11-62. 
BYDLOWSKI M. (b 2002), Les représentations inconscientes pendant la grossesse, La dette de vie. Itinéraire psychanalytique de la maternité. Paris, PUF, pp. 63-120.

CAHN R. (1987), «Approche métapsychologique du processus thérapeutique en institution de soins pour jeunes psychotiques », Perspectives psychiatriques, 26,8, pp.153-160.

GREEN A. (1983), "Le complexe de la mère morte ", Narcissisme de vie, narcissisme de mort, Paris, Editions de Minuit.

JENSEN J.S. et ZAHOUREK R., (1972), «Depression in mothers who have lost a newborn », Rocky Mountain medical journal, 71, 61-63.

LEWIS E. (1979), « Mourning by the family after stillbirth or neonatal death », Arch.dis.Child., 54, 303-306.

MACDOUGALL J. (1978), Plaidoyer pour une certaine anormalité, Paris, PUF.

MANZANO J., PALACIO-ESPASA F., ZILKHA N. (1999), Les scénarios narcissiques de la parentalité. Clinique de la consultation thérapeutique, Paris, PUF, Coll. « Le fil rouge ».

MILNER M. (1990), « Le rôle de l'illusion dans la formation du symbole. Les concepts psychanalytiques sur les deux fonctions du symbole », Journal de la psychanalyse de l'enfant, 8, pp. 244-278.

ROSENBERG B. (1997), « Le moi et son angoisse. Entre pulsion de vie et pulsion de mort », Monographies de la Revue Française de Psychana-lyse, Paris, PUF.

SQUIRES C. (2002), « Les psychothérapies périnatales lors d'une mort fœtale in utero : L'angoisse du clinicien face aux angoisses de mort. L'analyse du contretransfert. » In M. Bydlowski, C. Squires, D. Candilis (Eds), Recherche et interventions autour de la naissance, Editions E.S.F. VALABREGA J-P., (1980), Phantasme, mythe, corps et sens : une théorie psychanalytique de la connaissance, Paris, Science de l'homme, Payot.

WOLFF J., NELSON P., SHILLER P. (1970), « The émotional reaction to stillbirth », Amer J. of obstetrics and gyné., vol 108, n 1, 73-76.

ZEANAH C.H., " Adaptation following perinatal loss : a critical review », Journal of the american academy of child and adolescent psychiatry, 1989, 28, 467-480. 
